\title{
A RARE CASE OF SPONTANEOUS MITRAL LEAFLET PERFORATION LEADING TO SEVERE MITRAL REGURGITATION
}

\author{
Ashok Ari, Nemani Lalitha.
}

\begin{abstract}
Spontaneous mitral leaflet perforations are rare. We are reporting a 60 year old hypertensive female patient admitted with pulmonary edema with clinically short systolic murmur at apex without ischemic changes on ECG. There is no $\mathrm{h} / \mathrm{o}$ chest pain, fever, blunt trauma of chest. TTE no RWMA, good bi- ventricular function, a posteriorly directed eccentric moderate - severe mitral regurgitation, normal LA,LV, no vegetation or prolapsed. TEE after stabilization showed whole in body of AML at its midpoint leading to severe regurgitant jet in to LA with Vena contracta of $0.8 \mathrm{~mm}$. All the papillary muscles were intact and there is no prolapse of leaflets and no vegetation. Cardiac catheterization revealed increased LVEDP and PCWP with mild PAH with normal coronary arteries.

KEY WORDS: Spontaneous rupture, mitral leaflet.
\end{abstract}

\section{INTRODUCTION}

Mitral leaflet perforation is a rare cause of mitral regurgitation usually following infective endocarditis. Usually these patients are sick and requires urgent surgery. Other causes of mitral leaflet perforation are blunt trauma to chest, post mitral commissuratomy. In a stable patient at least we may get the past history of infective endocarditis. We are reporting case without the precipitating factors.

\section{CASE REPORT:}

A 60 year old female patient who is hypertensive, non DM presented to hospital with complaints of shortness of breath - class 3 and palpitations since 1 month. There is no $\mathrm{h} / \mathrm{o}$ chest pain, fever, blunt trauma of chest.

Article received on 25 March2016, published on 30 April 2016.

Ashok Ari ${ }^{1}$, Nemani Lalitha ${ }^{2}$.

${ }^{1}$ DM student, Department of Cardiology,NIMS,India

${ }^{2}$ Asst. Professor of Department of Cardiology

Corresponding author: Ashok Ari

Email: ashokari@gmail.com
On examination patient has tachycardia, normo-tension, elevated JVP with prominent V wave, grade 4/6 PSM in mitral area with $S_{3}$, prominent wheeze, bi-basal crepititions.

The complete blood count, electrolytes and liver function tests were within normal limits. The cardiac enzymes including troponin I and creatine kinase were also normal. The baseline electrocardiogram showed LVH. Chest x-ray demonstrated pulmonary edema.

Trans-thoracic echocardiography showed no RWMA, good bi-ventricular function, a posteriorly directed eccentric moderate - severe mitral regurgitation, mild dilated LA, LV, no vegetation or prolapsed (Fig 1, 2). After stabilization with vasodilators, diuretics, TEE was done which showed some interesting findings. Mid esophageal 4 chamber view showed isolated prolapse of A2 segment with rupture of AML at its midpoint leading to severe regurgitant jet in to LA. It is around 0.8 $\mathrm{mm}$ in length (Fig 3,4). All the papillary muscles were intact and there is no vegetation. Cardiac catheterization revealed increased LVEDP and PCWP with mild PAH. Coronary arteries were normal. Patient has sent for mitral valve repair.

\section{DISCUSSION}

There are case reports of rupture of mitral leaflets and papillary muscles secondary to ischemia, infective endocarditis, blunt trauma to chest, MVP, calciphylaxis of mitral leaflet with rupture in CKD patients. Most of these patients present in acute pulmonary edema due to sudden increase in LA pressures. Most of the cases we tie over the crisis with decongestive therapy, vasodilators and in some even intra aortic balloon pump. Ultimately requires mitral valve repair if 
possible or replacement if additional infective endocarditis complications are there.

But this is very rare to have spontaneous rupture on mitral leaflet, with out remote history of prolonged fever. This case, at least to our knowledge seems to be the first case of isolated prolapse of one segment of leaflet with spontaneous rupture presenting at this age group.

Fig 1- Trans-thoracic apical 4 chamber view showing eccentric mitral regurgitant jet

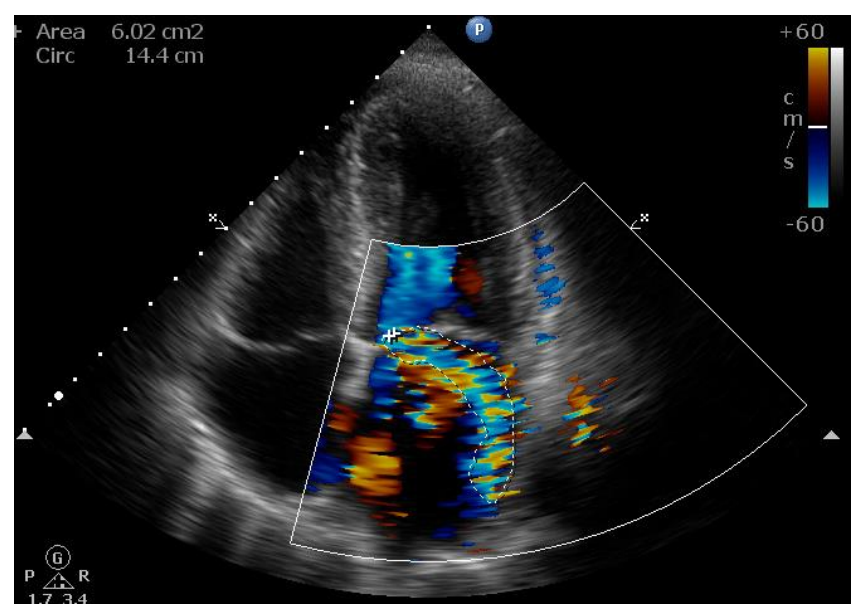

FIG 2- Parasternal long axis view showing posteriorly directed eccentric MR jet

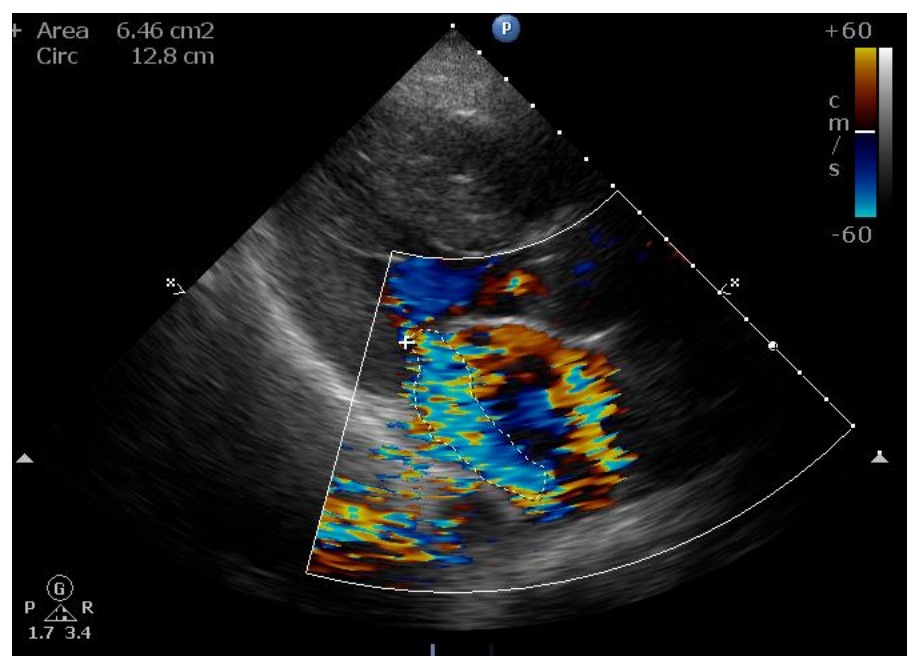

Fig 3 - TEE 4 chamber view with color interrogation showing defect in AML with mosaic jet in LA.

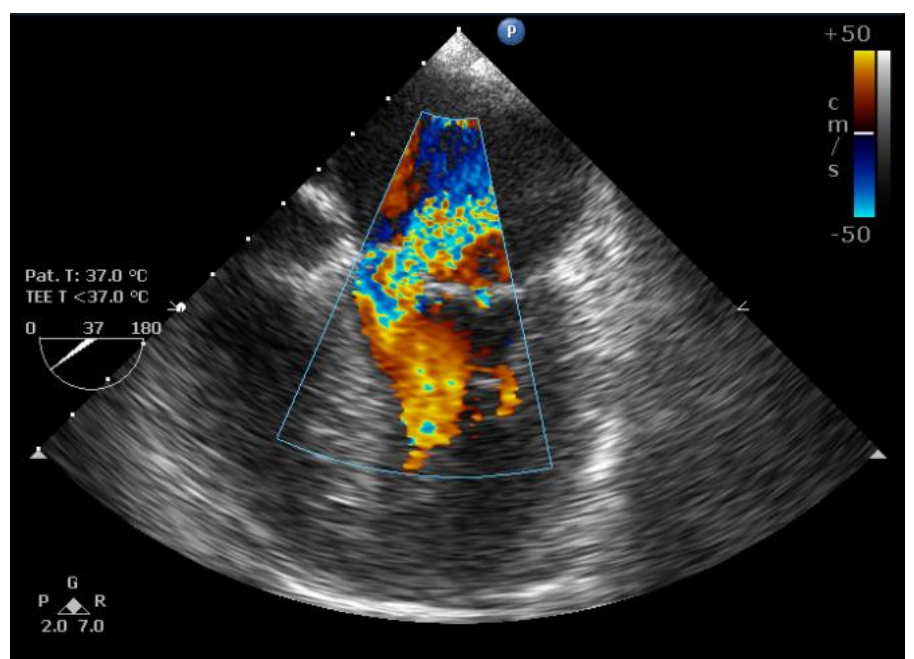

Fig 4- Mid Esophageal 2 Chamber View Showing Rupture In Mid Part Of AMLl

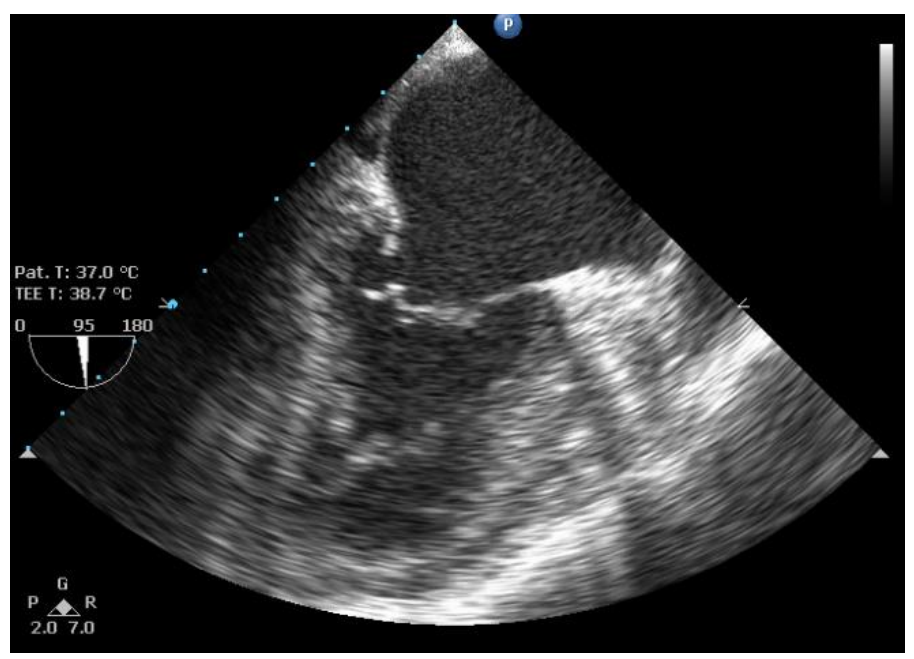

\section{REFERENCES}

1. Evans CF, DeFilippi CR, Shang E, Griffith BP, Gammie JS. Fresh autologous pericardium for leaflet perforation repair in mitral valve infective endocarditis.J Heart Valve Dis. 2013 Jul;22(4):560-6.

2. Vijay SK, Tiwari BC, Misra M, Dwivedi SK. Incremental value of three-dimensional transthoracic echocardiography in the assessment of ruptured aneurysm of anterior mitral leaflet. Echocardiography. 2014;31(1):E246. 
3. Javed U, Smith TW, Rogers JH. Percutaneous repair of anterior mitral leaflet perforation. J Invasive Cardiol. 2012 Mar;24(3):134-7.

4. Tsuchida H, Hirayama $T$, Yamaguchi $H$, Ishimaru S, Furukawa K, Takahashi M. A case of mitral regurgitation due to perforation of the mitral posterior leaflet. Nihon Kyobu Geka Gakkai Zasshi. 1990 Feb;38(2):303-6. Japanese. 